\title{
Characterization of Grafted Polybutadiene on a Poly(tetrafluoroethylene) Surface of Extremely Low Grafting Ratio
}

\author{
Katsuhiro Yamamoto, Shigetaka Shimada, ${ }^{\dagger}$ Masato SaKaguchi, $^{*}$ \\ and Yosiharu Tsujita \\ Nagoya Institute of Technology, Gokiso-cho, Showa-ku, Nagoya 466, Japan \\ *Ichimura Gakuen College, 61 Uchikubo, Inuyama 484, Japan
}

(Received November 18, 1996)

\begin{abstract}
Butadiene monomer (BD) was block copolymerized by the initiation of mechanoradicals formed by mechanical fracture of poly(tetrafluoroethylene) (PTFE) powder. Polybutadiene (PBD) propagating radicals are much more stable and much mobile above melting temperature of butadiene monomer. In order to determine extremely low grafting ratio, nitroxide free radicals produced by reactions of 2,6-dichloronitrosobenzene with double bonds in PBD on PTFE are quantitatively detected. The determination of $10^{-3}-10^{-1}(\%)$ of grafting ratio was possible by the spin labeling method and the grafted chains were regarded as isolated chains. The extremely low grafting ratio could not be detected by other methods. The estimated number average molecular weight of the grafted chain was strongly dependent on the pressure of BD monomer. KEY WORDS Grafted Polybutadiene / Poly(tetrafluoroethylene)/Characterization / Grafting Ratio / Isolated Chain / Electron Spin Resonance / Spin Labeling /
\end{abstract}

We have studied the structure and molecular motion of polymer chains tethered and adsorbed on poly(tetrafluoroethylene) (PTFE) ${ }^{1,2}$ and porous silica surfaces ${ }^{3,4}$ respectively, by the spin label method. Adsorbed polystyrene chains take "train" and "loop" or tail" conformations on the silica surface having large and small pore diameters, respectively. The structures of poly(ethylene oxide) chains on the silica are strongly dependent on the grafting ratio. ${ }^{5-7}$ The polyethylene (PE) alkyl radicals and peroxy radicals at the terminal of $\mathrm{PE}$ chains tethered on PTFE surface have high mobility even at $77 \mathrm{~K} .^{1,2}$ The high mobility was interpreted in terms of extremely low segmental concentration of PE molecules on the PTFE surface. These experimental findings elucidate the structure and molecular motion of isolated polymer chains, which are related to intra chain interactions and mechanism of aggregation of the isolated chains. Segmental density is the most important factor for the behaviors. Determination of both the grafting ratio and molecular weight of the grafted chains on the solid surface is indispensable for the evaluation of segmental concentration.

In this paper, we characterize grafted polybutadiene (PBD) on a PTFE surface by the spin labeling method. The stability of PBD propagating radicals and extremely low grafting ratio should be characteristic of isolated PBD chains on the PTFE surface.

\section{EXPERIMENTAL}

\section{Materials}

Poly(tetrafluoroethylene) (PTFE) (Fluon G-163, Asahi Glass Co., Ltd.) was used without purification. 1,3-Butadiene monomer (BD) (Tokyo Kasei Co., Ltd.) was purified by the freeze-pump-thaw method. 2,6Dichloro-nitrosobenzene (DCNB) was synthesized by oxidation of 2,6-dichloroaniline (Nacalai Tesque Co., Ltd.). ${ }^{9}$ Cis 1,4-polybutadiene was purchased from

\footnotetext{
† To whom correspondence should be addressed.
}

Polymer Products Inc. The polymer sample was purified three times by dissolving it in toluene and precipitating it in methanol and drying in a vacuum oven.

\section{Mechanical Fracture}

The mechanical fracture of the PTFE powder $(1.5 \mathrm{~g})$ with BD monomer was carried out by milling in a home-made vibration glass ball mill in vacuo at $77 \mathrm{~K} .^{1}$ The ESR sample tube connected to the glass ball mill was then placed in liquid nitrogen, and dropped into the ESR sample tube within $1 \mathrm{~s}$ by turning the glass ball mill upside down. After the allyl radicals at the chain end were observed, the sample was stored at room temperature till the grafting ratio was estimated.

(A) The BD monomer was introduced at pressures of 1, 7, 28, and 190 Torr into the PTFE samples and then the samples were milled for $24 \mathrm{~h}$ at $77 \mathrm{~K}$. After milling and ESR observation of the free radicals, non-reacted monomer was evacuated at $183 \mathrm{~K}$ for $1 \mathrm{~h}$ and stored at room temperature for a long time. The dry samples were coded as PTFE/PBD (R1), PTFE/PBD (R7), PTFE/PBD (R28), and PTFE/PBD (R190), respectively.

(B) BD monomer was introduced at pressures of 1, 7, 28, 70, 190, and 430 Torr into the PTFE samples and then the samples were milled for $24 \mathrm{~h}$ at $77 \mathrm{~K}$. After milling and ESR observation of the free radicals, the samples were stored at room temperature for a long time without removing the butadiene monomer. The wet samples were coded as PTFE/PBD (1), PTFE/PBD (7), PTFE/PBD (28), PTFE/PBD (70), PTFE/PBD (190), and PTFE/PBD (430), respectively.

\section{ESR Measurements}

ESR spectra at $77 \mathrm{~K}$ and higher temperatures were observed at low microwave power to avoid power saturation and with $100 \mathrm{kHz}$ fielded modulation using JEOL JES-FE3XG and JES-RE1XG spectrometers (X band) coupled to microcomputers (NEC PC 9801). The signal of diphenylpicrilhydrazil (DPPH) was used as the $g$-value standard. The magnetic field sweep was cali- 
brated with known splitting constants of $\mathrm{Mn}^{2+}$.

\section{RESULTS AND DISCUSSION}

\section{Identification of Free Radicals Produced by Mechanical Fracture of PTFE with Butadiene Monomer}

Figure 1(a) shows the ESR spectrum of free radicals produced by mechanical fracture of the PTFE/PBD (430). A narrow and well-resolved spectrum was observed at higher temperatures than melting temperature of BD, $164 \mathrm{~K}$. This narrow line width can be interpreted in terms of high mobility of the free radicals. The $6 \times 2$ lines are caused by large $(c a .1 .4 \mathrm{mT})$ and small $(c a$. $0.4 \mathrm{mT}$ ) hyperfine splittings (hfs) which seem due to five equivalent hydrogen nucleus and other one hydrogen nuclei, respectively. The values agree well with data obtained by Iwasaki et al. ${ }^{10}$ for allyl type chain end radicals, $-\mathrm{CH}_{2}-\mathrm{CH} \beta_{2}-\mathrm{CH} \alpha 3=\mathrm{CH} \alpha 2-\mathrm{CH} \alpha \mathrm{l}_{2}$.

Figure 1(b) shows the calculated spectrum assuming hfs values of $A_{\alpha 1}=1.34 \mathrm{mT}, A_{\alpha 2}=0.34 \mathrm{mT}, A_{\alpha 3}=1.49$ $\mathrm{mT}, A_{\beta}=1.35 \mathrm{mT}, g=2.00224$. The agreement between the simulated spectrum and observed spectrum was excellent, indicating the mechanoradicals to initiate the block copolymerization of butadiene monomer and the PBD propagating radicals to be stable at temperatures above melting temperature of $\mathrm{BD}$ monomer. The extremely narrow line width of the ESR spectrum in Figure 1(a) is caused by the high molecular mobility of the PBD propagating radicals.

Surprisingly, the narrow line width is of the same order of magnitude as those of the spectra of propagating radicals in the solution. In general, the narrow ESR spectra are observed simultaneously with generation of free radicals during polymerization as the propagating radicals are unstable. For example, well-resolved spectra due to $\mathrm{PB}$ propagating radicals were observed recently by Kamachi et al. ${ }^{11}$ Matsumoto et al. synthesized many

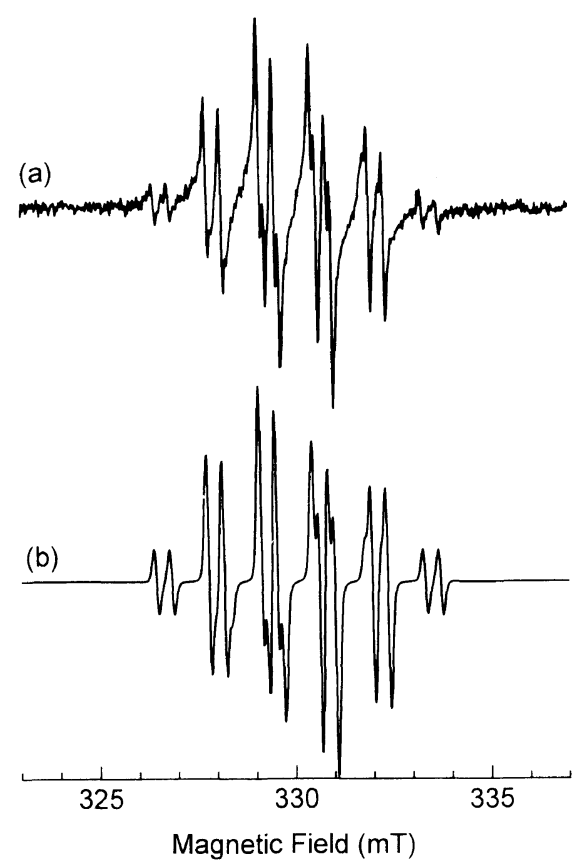

Figure 1. Comparison of observed ESR spectrum (a) with calculated spectrum (b) of PBD propagating radicals on PTFE surface. Measurements were made at $193 \mathrm{~K}((\mathrm{PTFE} / \mathrm{PBD}((430))$. monomers, polymerization of which produced propagating radicals with chemically stable structures and narrow spectra in toluene solution. ${ }^{12}$

The propagating PBD radicals tethered on the PTFE surface, were much more stable and mobile. The segmental concentration of the grafted chains would thus appear extremely low.

\section{Decay of PBD Radicals on the PTFE Surface}

To confirm the stability of PBD propagating radicals, an elaborate experiment of annealing was made as follows. After observing the spectrum of the milled sample at $77 \mathrm{~K}$, the sample was annealed at a desired temperature for $15 \mathrm{~min}$, quenched at $77 \mathrm{~K}$ and ESR measurements were made at the same temperature. The procedure was repeated many times with increasing annealing temperatures. Figure 2 shows the decay curves of PBD propagating radicals in the wet samples of PBD grafted on PTFE. The propagating radicals were much more stable at higher temperatures than melting temperature of $\mathrm{BD}$ monomer, $164 \mathrm{~K}$. The free radicals trapped in all samples did not decay below $183 \mathrm{~K}$ and ca. $30 \%$ of free radicals trapped in the PTFE/PBD (7) sample were still alive at $255 \mathrm{~K}, 90 \mathrm{~K}$ higher than the melting temperature.

One of the authors studied in detail the mechanism of the decay of free radicals trapped in solid polymers. ${ }^{13}$ The decay should be analyzed by diffusion-controlled bimolecular reaction. "Diffusion" means mass translation and/or the migration of radical sites in threedimensional space. The free radical migration is caused by repeated hydrogen abstraction of the radicals. The probability of the inter molecular migration should increase with molecular density. The decay rate of PBD propagating radicals was strongly dependent on the pressure of BD monomer as shown in Figure 2. The mass translation of the PBD chains grafted on the PTFE surface should be inhibited because one end of the grafted chain is fixed to the surface.

The segmental concentrations of the grafted PBD chains should thus be extremely low so that propagating radicals would not abstract fluorine and hydrogen atoms

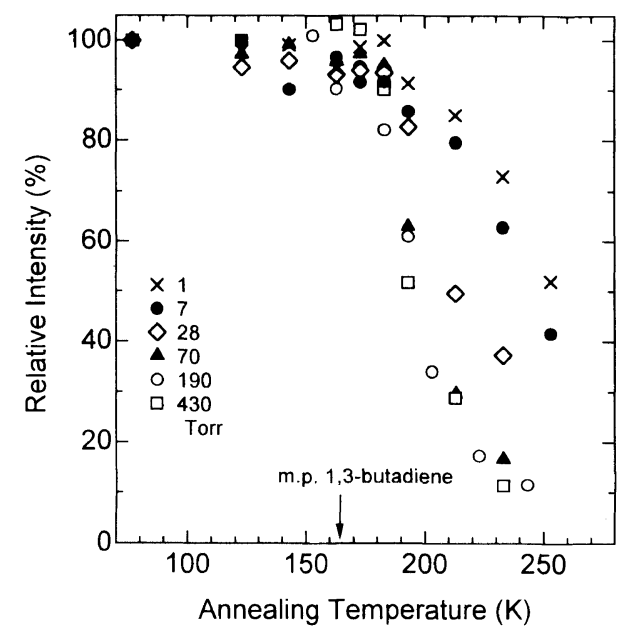

Figure 2. Decay curves of PBD propagating radicals on PTFE surfaces. $\times$, PTFE/PBD (1);, PTFE/PBD (7); $\diamond$, PTFE/PBD (28); $\Delta$, PTFE/PBD (70); O, PTFE/PBD (190); $\square$, PTFE/PBD (403). ESR measurements were made at $77 \mathrm{~K}$. 
easily in spite of high mobility.

\section{Determination of Extremely Low Grafting Ratio}

Weight increase due to copolymerization for the samples of PBD grafted on PTFE could not be detected by gravimetric measurement or pyrolysis weight loss. This suggests that the samples have extremely low grafting ratios.

Rockenbauer et al. ${ }^{14-16}$ found that nitroxide free radicals were produced by reactions of spin labeling reagents with many organic compounds. The mechanism of the reaction of 2,6-dichloronitrosobenzene with double bonds in PBD is as follows.

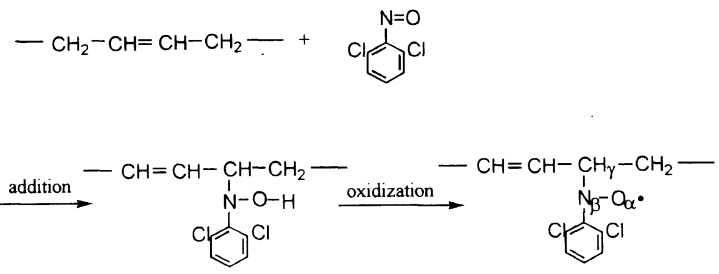

It may be considered that the amounts of the produced nitroxide radicals should be a function of the number of double bonds in a sample. Figure 3 shows ESR spectrum of free radicals in toluene solution of cis 1,4polybutadiene and 2,6-dichloronitrosobenzene. The ESR spectrum (b) for the spin label connected to the polymer grafted on the PTFE surface is just the same as that (a) for the spin-label to the cis 1,4-polybutadiene. Hfs due to nitrogen $(1.28 \mathrm{mT})$ and $\gamma$ hydrogen $(0.758 \mathrm{mT}) \mathrm{nu}-$ cleus and $g$-value $=2.0059$ agree with those for polybutadiene, obtained by Rockenbauer et al. ${ }^{16}$ The intensity (I) of the ESR spectra for the spin-labels connected to the cis 1,4 PBD sample increases, has a maximum, and decreases with increasing storage time at room temperature as shown in Figure 4. This suggests that the decay of the free radicals occurs with the generation

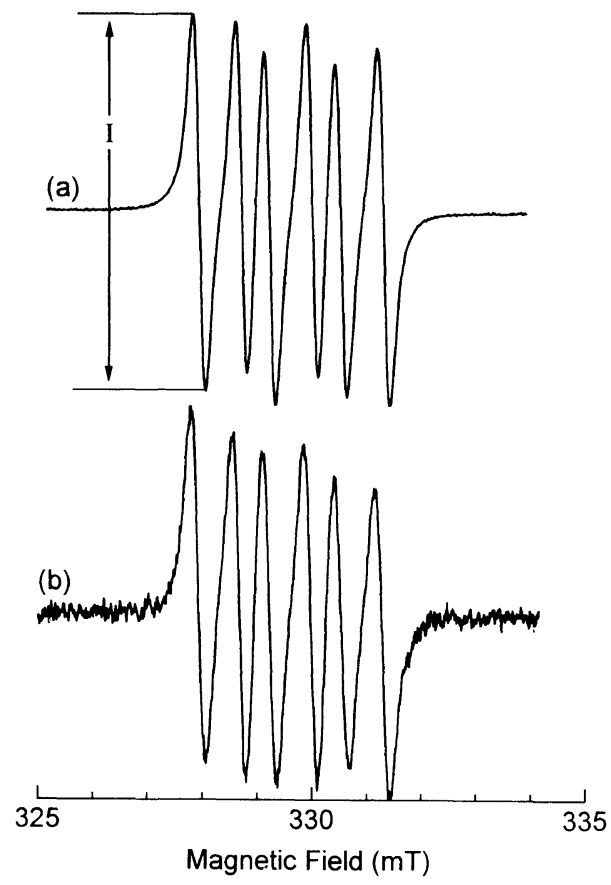

Figure 3. ESR spectra of spin-labeled PBD in toluene solution: (a), cis 1,4-polybutadiene; (b), polybutadiene grafted on PTFE surface (PTFE/PBD (430)). Measurements were made at room temperature. of the radicals. The relative intensity and the maximum value of the intensity increase with the amount of PBD in the ESR sample tube. Figure 5 shows the absolute amount of PBD as a function of maximum concentration of spin-labels in toluene solution, obtained from the data in Figure 4. This should serve as a calibration curve for the determination of the absolute amount of PBD in a sample. Figure 6 shows variation of ESR intensity of spin-labels in PBD chains grafted on PTFE with storage time. ESR intensity was maximum around $2000 \mathrm{~min}$ of storage time and decreased with time. The maximum or asymptotic value also increased with pressure of BD monomer. The grafting ratio can be determined from the maximum (asymptotic) value using the calibration curve (Figure 5). Figure 7 shows plots of the grafting ratio for the grafted samples against the pressure of BD monomer. The extremely low grafting ratio, $1.8 \times 10^{-3}-5.0 \times 10^{-2} \%$ was estimated from the spin labeling method. The grafting ratio increased with pressure of the BD monomer and the grafting ratios for the wet samples exceeded those of the dried samples. These facts suggest that the PBD radicals propagate along

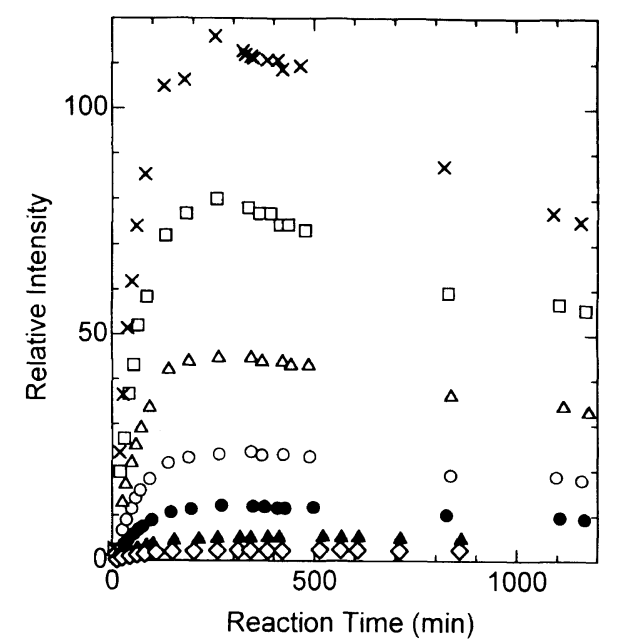

Figure 4. Relative concentration (relative intensity of ESR spectra) of spin-labeled PBD in toluene solution versus reaction time. Reference sample of cis 1,4-polybutadiene was diluted at $13.5(\times), 6.78(\square), 3.39$ $(\triangle), 1.69(\bigcirc), 0.797(\bigcirc), 0.398(\Delta)$, and $0.199(\diamond)\left(\mathrm{gl}^{-1}\right)$.

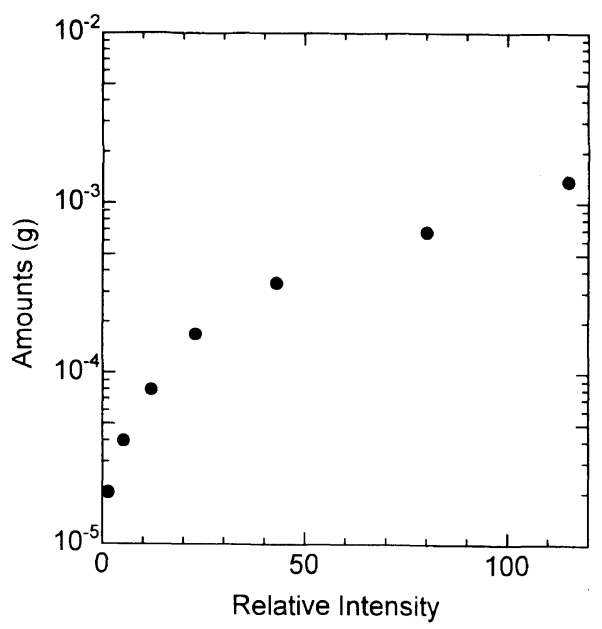

Figure 5. Absolute amount of polybutadiene in the sample tube versus maximum (asymtotic) concentration (relative intensity of ESR spectra) of spin-label in toluene solution, indicated in Figure 4. 


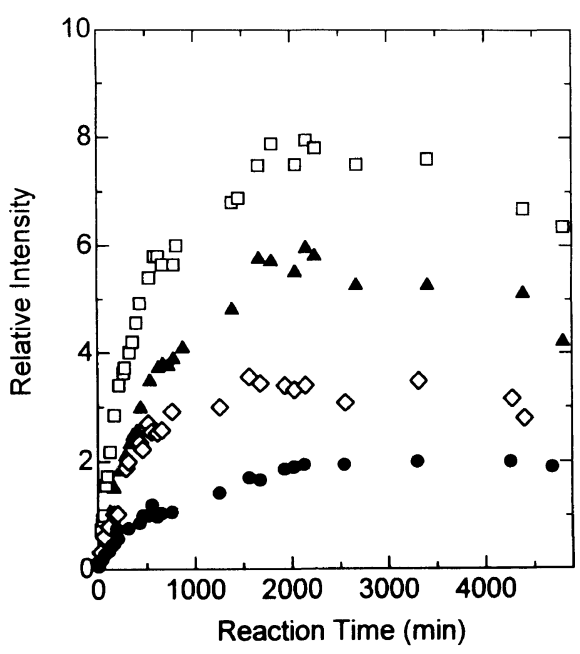

Figure 6. Variation of concentration (relative intensity of ESR spectra) of spin-labeled PBD on the PTFE surface in toluene solution with reaction time. The arbitrary unit is just the same as that in Figure 4. $\square$, PTFE/PBD (R 190); $\triangle$, PTFE/PBD (R28); $\diamond$, PTFE/PBD (R7); ๑, PTFE/PBD (R1).

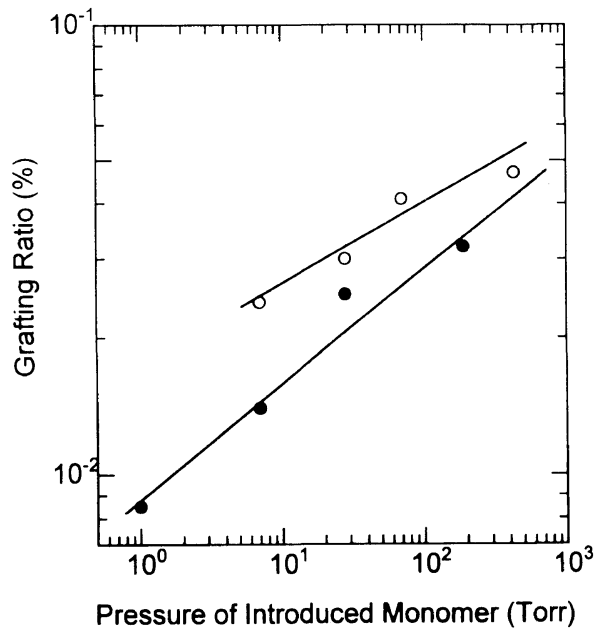

Figure 7. Plots of grafting ratios for the PTFE/PBD samples against pressure of BD monomer: $\boldsymbol{O}$, non-reacted monomer was removed at $183 \mathrm{~K}$ for $1 \mathrm{~h}$ after milling and sample was stored at room temperature for a long time; $O$, sample was stored at room temperature for a long time without removing non-reacted monomer after milling.

the chain and the molecular weight of the grafted PBD increases with amount of $\mathrm{BD}$ monomer. Because, the concentration of mechanoradicals of PTFE as an initiation reagent of copolymerization was fixed for all samples and all the mechanoradicals were converted to the PBD radicals during mechanical fracture at $77 \mathrm{~K}$. Nonreacted $\mathrm{BD}$ monomer in the wet sample reacted with PBD propagating radicals and the degree of polymerization increased above $183 \mathrm{~K}$, at which the BD monomer in the dried samples was removed.

The tethered points on the PTFE surface are estimated to be $6.8 \times 10^{16} \mathrm{~g}^{-1}$ from the absolute concentration of mechanoradicals produced by the mechanical fracture. $100 \%$ of mechanoradicals initiated BD monomer and produced grafted PBD chains on the PTFE surface. Therefore, the degree of polymerization of the
Table I. Grafting ratios and degree of polymerization of polybutadiene on the PTFE surface

\begin{tabular}{lcc}
\hline Samples & Grating ratio/\% & $\begin{array}{c}\text { Degree of } \\
\text { polymerization }\end{array}$ \\
\hline PTFE/PBD (R1) & $8.5 \times 10^{-3}$ & 14 \\
PTFE/PBD (R7) & $1.4 \times 10^{-2}$ & 23 \\
PTFE/PBD (R28) & $2.5 \times 10^{-2}$ & 41 \\
PTFE/PBD (R190) & $3.2 \times 10^{-2}$ & 52 \\
PTFE/PBD (7) & $2.4 \times 10^{-2}$ & 39 \\
PTFE/PBD (28) & $3.0 \times 10^{-2}$ & 49 \\
PTFE/PBD (70) & $4.1 \times 10^{-1}$ & 67 \\
PTFE/PBD (430) & $4.7 \times 10^{-2}$ & 77 \\
\hline
\end{tabular}

grafted PBD can be easily calculated as indicated in Table I.

The area per tethered point on the PTFE surface $\left(3.1 \times 10^{3} \AA /\right.$ point $)$ was found from the tethered points $\left(6.8 \times 10^{16} \mathrm{~g}^{-1}\right)$ and the specific area of the PTFE powder $\left(2.1 \mathrm{~m}^{2} \mathrm{~g}^{-1}\right)$. We can concluded that the grafted PBD chains of low polymerization should be regarded as isolated chains which cannot contact with each other.

We will study in detail the structures and molecular motion of the PBD isolated chains grafted on the PTFE surface and publish the results in the next paper.

\section{CONCLUSIONS}

Characteristics of PBD chains grafted on the PTFE surface by mechanical fracture of PTFE with BD monomer. PBD propagating radicals are much more stable above the melting temperature of $\mathrm{BD}$ monomer. PBD chains are much more mobile at low temperatures. The grafting ratio is extremely low. PBD chains may thus be regarded as isolated chains.

\section{REFERENCES}

1. M. Sakaguchi, T. Yamaguchi, S. Shimada, and Y. Hori, Macromolecules, 26, 2612 (1993).

2. S. Shimada, A. Suzuki, M. Sakaguchi, and Y. Hori, Macromolecules, 29, 973 (1996)

3. S. Shimada, A. Sugimoto, and M. Kawaguchi, Polymer, 37, (1996) in press.

4. S. Shimada, Y. Hane, and T. Watanabe, Polymer, 38 (1997), to be accepted.

5. S. Shimada and T. Watanabe, Polymer, 38 (1997), to be submitted.

6. S. Shimada and T. Watanabe, Polymer, 38 (1997), to be submitted.

7. T. Tajouri and H. Bouchri, Polymer, 37, 3185 (1996).

8. K. Yamamoto, S. Shimada, M. Sakaguchi, and Y. Tsujita, Macromolecules, 30 (1997), in press.

9. R. R. Hones and P. R. Bayer, J. Am, Chem. Soc., 82, 3454 (1960).

10. T. Ohmori, T. Ichikawa, and M. Iwasaki, Bull. Chem. Soc. Jpn., 46, 1383 (1973).

11. M. Kamachi and A. Kajiwara, Macromolecules, 29, 2378 (1996).

12. A. Matsumoto and B. Giese, Macromolecules, 29, 3758 (1996).

13. S. Shimada, Prog. Polym. Sci., 17, 1045 (1993).

14. M. Gyor, A. Rockenbauer, and F. Tudos, Tetrohedron Lett., 27, 3759 (1986)

15. M. Gyor, A. Rockenbauer, and F. Tudos, Tetrohedron Lett., 27, 4759 (1986)

16. M. Gyor, A. Rockenbauer, and F. Tudos, Macromolecules, 20, 3083 (1987) 